SignóticA 


\section{Universidade Federal de Goiás}

REITOR

Orlando Afonso Valle do Amaral

VICE-REITOR

Manoel Rodrigues Chaves

Pró-Reitora de Pesquisa e Inovação

Maria Clorinda Soares Fioravanti

Pró-Reitor de Pós-Graduação

José Alexandre F. Diniz Filho

Diretor do Cegraf/UFG

Antonio Corbacho Quintela

\author{
Diretor da Faculdade de Letras \\ Francisco José Quaresma Figueiredo \\ Coordenadora do Programa de \\ Pós-Graduação em Letras e Linguística \\ Joana Plaza Pinto \\ Editora Geral \\ Joana Plaza Pinto \\ Editor da Área de Estudos LiteráRIos \\ Wilson José Flores Junior \\ Solange Fiúza Cardoso Yokozawa \\ Editora da Área de Estudos Linguísticos \\ Rosane Rocha Pessoa
}

\section{Conselho Editorial da Área de Estudos Linguísticos}

Ana Cristina Ostermann, Universidade do Vale do Rio dos Sinos, São Leopoldo-RS, Brasil; Ana Maria Stahl Zilles, Universidade do Vale do Rio dos Sinos, São Leopoldo-RS, Brasil; Anna Chistina Bentes, Universidade Estadual de Campinas, Campinas-SP, Brasil; Branca Falabella Fabricio, Universidade Federal do Rio de Janeiro, Rio de Janeiro-RJ, Brasil; Claudiana Nogueira de Alencar, Universidade Estadual do Ceará, Fortaleza-CE, Brasil; Candida Soares da Costa, Universidade Federal de Mato Grosso, Cuiabá-MT, Brasil; Carmen Caldas-Coulthard, University of Birmingham, Inglaterra, Reino Unido; Daniel do Nascimento e Silva, Universidade Federal do Rio de Janeiro-RJ, Brasil; Débora de Carvalho Figueiredo, Universidade Federal de Santa Catarina, Florianópolis-SC, Brasil; Dilys Karen Rees, Universidade Federal de Goiás, Goiânia-GO, Brasil; Djane Antonucci Correa, Universidade Estadual de Ponta Grossa, Ponta Grossa-PR, Brasil; Eliane Marquez da Fonseca Fernandes, Universidade Federal de Goiás, Goiânia-GO, Brasil; Guilherme Veiga Rios, Universidade de Brasília, Brasília-DF, Brasil; John Robert Schmitz, Universidade Estadual de Campinas, Campinas-SP, Brasil; José Esteban Hernández, University of Texas, Edinburg-TX, Estados Unidos da América; Kanavillil Rajagopalan, Universidade Estadual de Campinas, CampinasSP, Brasil; Kassandra da Silva Muniz, Universidade Federal de Ouro Preto, Mariana-MG, Brasil; Kátia Menezes de Sousa, Universidade Federal de Goiás, Goiânia-GO, Brasil; Leo Wetzels, Free University of Amsterdam, Amsterdam, Holanda; Maria Bernadete Fernandes de Oliveira, Universidade Federal do Rio Grande do Norte, Natal-RN, Brasil; Maria Izabel Santos Magalhães, Universidade Federal do Ceará, Fortaleza-CE, Brasil; Maria Inês Pagliarini Cox, Universidade Federal de Mato Grosso, Cuiabá-MT, Brasil; Maria Viviane do Amaral Veras, Universidade Estadual de Campinas, Campinas-SP, Brasil; Monica Heller, Ontario Institute for Studies in Education, University of Toronto, Canadá; Piia K. Varis, University of Tilburg, Tilburg, Holanda; Raquel Bello Vázquez, Universidad de Santiago de Compostela, Santiago, Espanha; Roland Schmidt-Riese, Katholische Universität Eichstätt-Ingolstadt, Eichstätt, Baviera, Alemanha; Silvia Lúcia Bigonjal Braggio, Universidade Federal de Goiás, Goiânia-GO, Brasil; Terezinha de Jesus Machado Maher, Universidade Estadual de Campinas, Campinas-SP, Brasil; Vera Lúcia Menezes de Oliveira Paiva, Universidade Federal de Minas Gerais, Belo Horizonte-MG, Brasil; Wilmar da Rocha D'Angelis, Universidade Estadual de Campinas, Campinas-SP, Brasil.

\section{Conselho Editorial da Área de Estudos Literários}

Affonso Romano de Sant'anna, Pontifícia Universidade Católica do Rio de Janeiro (PUC-Rio), Rio de Janeiro, RJ, Brasil; António Apolinário Lourenço, Universidade de Coimbra, Coimbra, Portugal; Antonio Donizeti Pires, Universidade Estadual Paulista Júlio de Mesquita Filho, Araraquara, SP, Brasil; Arnaldo Saraiva, Universidade do Porto, Porto, Portugal; Edvaldo Bergamo, Universidade de Brasília (UnB), Brasília, DF, Brasil; Fernando Fábio Fiorese Furtado, Universidade Federal de Juiz de Fora (UFJF), Juiz de Fora, MG, Brasil; Heleno Godói, Universidade Federal de Goiás (UFG), Goiânia, GO, Brasil; Jacqueline Penjon, Univerdade de Paris III, Sorbonne-Nouvelle, Paris, França; Jorge Alves Santana, Universidade Federal de Goiás (UFG), Goiânia, GO, Brasil; Luiz Roberto Velloso Cairo, Universidade Estadual Paulista Júlio Mesquita Filho (UNESP), Assis, SP, Brasil; Maria Laura Bettencourt Pires, Universidade Católica Portuguesa, Lisboa, Portugal; Paulo Franchetti, Universidade Estadual de Campinas (Unicamp), Campinas, SP, Brasil; Pedro Carlos Louzada Fonseca, Universidade Federal de Goiás (UFG), Goiânia, GO, Brasil; Regina Zilberman, Universidade Federal do Rio Grande do Sul (UFRGS), Porto Alegre, RS; Roberto Samartim, Universidad da Corunha, Corunha, Espanha; Sandra Margarida Nitrini, Universidade de São Paulo (USP), São Paulo, SP, Brasil; Wladimir Krysinski, Université de Montréal, Montréal, Canadá; Zahidé Lupinacci Muzart, Universidade Federal de Santa Catarina (UFSC), Florianópolis, SC, Brasil; Zênia de Faria, Universidade Federal de Goiás (UFG), Goiânia, GO, Brasil. 
ISSN versão on-line 2316-3690

ISSN versão impressa 0103-7250

Universidade Federal de Goiás

\section{SignÓticA \\ Revista do Programa de Pós-Graduação \\ em Letras E Linguística/FACULdAde DE Letras}

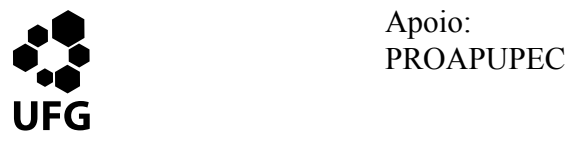

Signótica, Goiânia, v. 28, n. 1, jan./jun. 2016 
Signótica: revista do Programa de Pós-Graduação em Letras e Linguística/ Faculdade de Letras - Goiânia: Programa de Pós-Graduação em Letras e Linguística/Faculdade de Letras, 2014.

Semestral a partir do v. 15 .

Descrição baseada em: v. 28, n. 1 (jan./jun. 2016).

ISSN versão on-line 2316-3690

ISSN versão impressa 0103-7250

1. Universidade Federal de Goiás. Faculdade de Letras. Programa de Pós-Graduação em Letras e Linguística.

CDU 8(05)

REVISÃo

Português

Bruna Mundim Tavares

Fabiene Riâny Azevedo Batista

Janaynne Carvalho do Amaral

Pedro Augusto de Lima

INGLÊS

Pedro Augusto de Lima

ESPANHOL

Bruna Mundim Tavares

Sara Guiliana G. Belaonia
EDITORAÇÃo

Ricardo Campos

PAdRonizaÇÃo EdITORIAL:

Divisão de Periódicos - CEGRAF/UFG

Apolo ESPECIAL:

Programa de Apoio às Publicações Científicas da UFG

Endereço para correspondência:

Signótica

Caixa Postal 131

Telefone: 5562 3521-1136

74001-970 - Goiânia-GO

Endereço eletrônico: www.revistas.ufg.br/index.php/sig

E-mail: signotica@gmail.com 
A MANIFESTA FICCIONALIDADE E A ESTRUTURA TRIÁDICA EM “A HORA E VEZ de Augusto Matraga” DÉBORA FERRI

ESCREVIVÊNCIA E PRODUÇÃO DE SUBJETIVIDADES: REFLEXÕES EM TORNO DE “OlHos D’ÁGUA”, DE Conceição Evaristo

Henrique Furtado de MELO

Maria Carolina de GODOY

CARMEN EM PERSPECTIVA DIALÓGICA: REVERBERAÇÕES INQUIETAS DE UM PROCESSO DE CONSTRUÇÃO DRAMATÚRGICO-PERFORMATIVA NO CONTEXTO UNIVERSITÁRIO

Jean Carlos GONÇALVES

MichelLE BoCCHI GONÇALVES

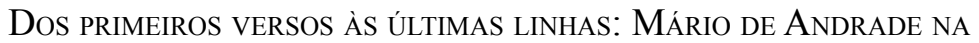

TRAJetória literária de Pedro NaVA

Júlio de Souza Valle NETO. 61

A PONTUAÇÃo COMO RECURSO DE ESTILO NO MINICONTO “FOSSO DO SOM”, DE JoÃo GilBerto Noll

LEANDRO PASSOS 81

De Tempo e Eternidade À INVENÇÃo de Orfeu, Jorge de Lima em Busca DO TEMPO ORIGINAL

Luciano Marcos Dias CAVALCANTI 99

ESTUDO DAS FONTES DE O SAMBA RURAL PAULISTA:

TRADIÇÃO E VARIANTES AUTORAIS

Manoel Mourivaldo Santiago ALMEIDA

Mario SANTIN FRUGIUELE 125 
ENTRE LIVROS, FLORESTAS E DESEJOS:

A FORMAÇÃo LITERÁRIA DO JOVEM NERUDA

Marcelo Ferraz de PAULA .................................................................. 143

AMOR, EROTISMO E PAIXÃO EM FLORBELA ESPANCA

Marly CATARINA SOARES 175

CONSIDERAÇÕES SOBRE CRÍTICA DE POESIA CONTEMPORÂNEA

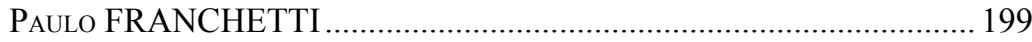

NoVE, NOVENA E A ESCRITA EM “O PÁSSARo TRANSPARENTE”, De OSMAN Lins

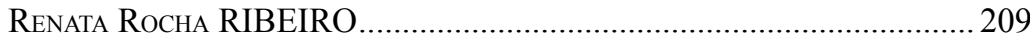


THE STATED FICTIONALITY AND THE TRIADIC STRUCTURE IN “A HORA E VEZ DE Augusto Matraga”

DÉBORA FERRI 1

ESCREVIVENNCIA AND SUBJECTIVITY PRODUCTION: REFLECTIONS AROUND “OlHos D’ÁGUA”, By Conceição Evaristo

Henrique Furtado de MELO

Maria Carolina de GODOY

CARMEN IN DIALOGICAL PERSPECTIVE: RESTLESS REVERBERATIONS OF A DRAMATURGICAL-PERFORMATIVE CONSTRUCTION PROCESS IN THE UNIVERSITY CONTEXT

Jean Carlos GOnÇAlves

Michelle Bocchi GONÇALVES

From the early verses to the late prose: Mário de ANdrade in the Literary CAReER of Pedro NaVA

Júlio de Souza Valle NETO

PUNCTUATION AS A STYLISTIC RESOURCE IN THE SHORT-SHORT STORY "FOSSO DO SOM”, BY JoÃo GilberTo Noll

LEANDRO PASSOS 81

From Tempo e Eternidade to InVENÇÃo de OrFeU, Jorge de Lima in SEARCH OF THE ORIGINAL TIME

Luciano Marcos Dias CAVAlCANTI .99

STUDY OF THE SOURCES OF O SAMBA RURAL PAULISTA:

TRADITION AND AUTHOR'S VARIANTS

Manoel Mourivaldo Santiago ALMEIDA

Mario SANTIN FRUGIUELE 125 
BETWEEN BOOKS, FORESTS AND WISHES:

THE LITERARY FORMATION OF YOUNG NERUDA

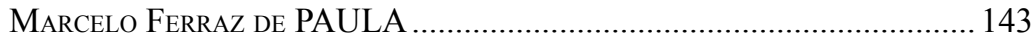

LOVE, EROTICISM AND PASSION IN FLORBELA ESPANCA

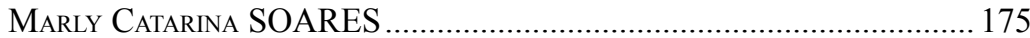

CONSIDERATIONS ON CONTEMPORARY POETRY CRITICISM

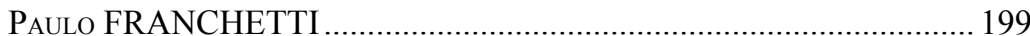

NINE, NOVENA AND WRITING IN “THE TRANSPARENT BIRD”, BY OSMAN LiNS

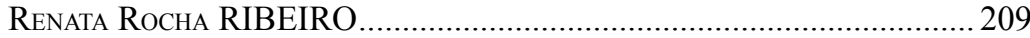


LA FICCIONALIDAD DECLARADA Y LA ESTRUCTURA TRIÁDICA EN “A HORA E Vez de Augusto Matraga"

DÉBORA FERRI

ESCREVIVÊNCIA Y PRODUCCIÓN DE SUBJETIVIDADES: REFLEXIONES SOBRE “OlHos D’ÁGUA”, DE Conceição Evaristo

Henrique Furtado de MELO

Maria Carolina de GODOY

CARMEN EN PERSPECTIVA DIALÓGICA: REVERBERACIONES INQUIETAS DE UN PROCESO DE CONSTRUCCIÓN DRAMATÚRGICO-PERFORMATIVA EN EL ÁMBITO UNIVERSITARIO

JeAn Carlos GONÇALVES

Michelle Bocchi GONÇALVES

Desde los PRIMERos Versos hasta las Últimas líNeas: MáRIO de Andrade en la carrera literaria de Pedro Nava

JÚlio de Souza Valle NETO 61

LA PUNTUACIÓN COMO RECURSO DE ESTILO EN EL MINICUENTO “FOSSO DO SOM” DE JoÃo GilberTo NOLL

LEANDRO PASSOS

De Tempo e Eternidade a la INVENÇÃo de ORFEU, Jorge de Lima en BUSCA DEL TIEMPO ORIGINAL

Luciano Marcos Dias CAVALCANTI .99

ESTUDIO DE LAS FUENTES DE O SAMBA RURAL PAULISTA:

TRADICIÓN Y VARIANTES DE AUTOR

Manoel Mourivaldo Santiago AlMEIDA

Mario SANTIN FRUGIUELE 125 
ENTRE LIBROS, BOSQUES Y DESEOS:

LA FORMACIÓN LITERARIA DEL JOVEN NERUDA

Marcelo Ferraz de PAULA ................................................................. 143

AMOR, EROTISMO Y PASIÓN EN FLORBELA ESPANCA

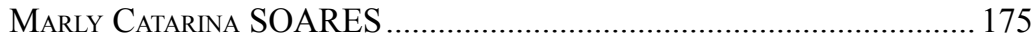

CONSIDERACIONES SOBRE CRÍTICA DE LA POESÍA CONTEMPORÁNEA

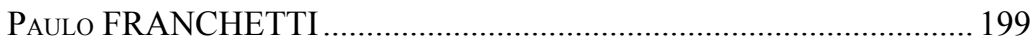

Nove, NoVENa y La escrita en “O PÁssaro transparente”, DE Osman Lins

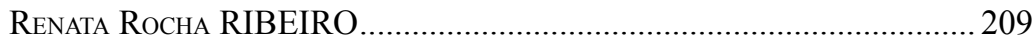

